\title{
Using surface impedance for calculating wakefields in flat geometry
}

\author{
Karl Bane* and Gennady Stupakov \\ SLAC National Accelerator Laboratory, Menlo Park, California 94025, USA
}

(Received 20 January 2015; published 18 March 2015)

\begin{abstract}
Beginning with Maxwell's equations and assuming only that the wall interaction can be approximated by a surface impedance, we derive formulas for the generalized longitudinal and transverse impedance in flat geometry, from which the wakefields can also be obtained. From the generalized impedances, by taking the proper limits, we obtain the normal longitudinal, dipole, and quad impedances in flat geometry. These equations can be applied to any surface impedance, such as the known dc, ac, and anomalous skin models of wall resistance, a model of wall roughness, or one for a pipe with small, periodic corrugations. We show that, for the particular case of dc wall resistance, the longitudinal impedance obtained here agrees with a known result in the literature, a result that was derived from a very general formula by Henke and Napoly.

DOI: 10.1103/PhysRevSTAB.18.034401

PACS numbers: 74.25.nn, 29.27.-a, 41.60.-m, 84.37.+q
\end{abstract}

\section{INTRODUCTION}

In linac-based $\mathrm{x}$-ray free electron lasers (FELs), such as LCLS-II at SLAC, electron bunches are accelerated to energies on the order of GeV's and compressed to lengths of tens of microns before entering an undulator for lasing. The undulator chamber typically has a small aperture (a $5 \mathrm{~mm}$ vertical aperture in the case of LCLS-II), and the resistive wall wakefields induced in the chamber can be strong and result in significant head-to-tail energy variation; this, in turn, can negatively affect the lasing process. Thus, it is important to be able to perform accurate, short-range wakefield calculations in order to predict the FEL performance.

The cross section of the LCLS-II undulator beam pipe has a racetrack shape, with a smaller vertical than horizontal aperture, and in terms of the wakefield effects can be well approximated by two parallel plates which we designate as "flat" geometry. In a cylindrical pipe ("round" geometry) the calculation of the resistive wall (rw) high frequency impedances, or equivalently, the short-range wakefields has long been well understood [1,2]. In flat geometry the long-range rw wake-where by long range we mean long compared to the characteristic distance $s_{0}$, which in the LCLS-II undulator case is $\sim 10 \mu \mathrm{m}$-is also well understood [3]. For the case of arbitrary bunch lengths in flat geometry, Henke and Napoly have obtained a general solution for the longitudinal and transverse rw impedances [4]. However, under the assumption of normal metallic walls, it was shown in Ref. [5] that their solution for longitudinal impedance can be greatly simplified.

\footnotetext{
*Corresponding author. kbane@slac.stanford.edu

Published by the American Physical Society under the terms of the Creative Commons Attribution 3.0 License. Further distribution of this work must maintain attribution to the author $(s)$ and the published article's title, journal citation, and DOI.
}

In this paper, beginning with Maxwell's equations and assuming only that the wall interaction can be approximated by a surface impedance, we derive a formula for the generalized longitudinal impedance in flat geometry. By "generalized" we mean that the transverse positions of the driving and test charges can be located anywhere between the two plates. Note that Piwinski has derived generalized impedances for round and flat geometries, but his results are limited to low frequencies [3,6]. The generalized impedance allows one to consider situations such as when a beam is mis-steered far from the axis, or when a beam is spread over an aperture as sometimes is the case with collimators. In this paper we next show that for the special case where both particles are located near the axis, where the impedance is normally defined, the longitudinal impedance agrees with the previously obtained result of Ref. [5]. Next we obtain the generalized transverse impedances, and also the normal quadrupole and dipole impedances as limits as the driving and test particles approach the axis. In this paper, as specific examples, we generate plots of the normal impedances and wakes for the special case where the surface impedance represents resistive walls with dc conductivity. The paper ends with conclusions.

In this paper most calculations are performed in Gaussian units. To convert an impedance or wake to MKSA (meters, kilograms, seconds, amperes) units, one needs to multiply the cgs expression by $Z_{0} c / 4 \pi$, with $Z_{0}=377 \Omega$.

\section{A. Round geometry}

Consider a sinusoidally oscillating beam with wave number $k$ moving along the axis, in the $+z$ direction, at the speed of light $c$ in a round metallic pipe. The interaction with the resistance in the walls can be characterized by the surface impedance $Z_{s}(k) \equiv Z_{0} E_{z} / H_{\phi}$ or equivalently by the dimensionless surface impedance, $\zeta(k)=Z_{s} / Z_{0}=$ $E_{z} / H_{\phi}$. Here $Z_{0}=4 \pi / c$ is the impedance of free space, and $E_{z}$ and $H_{\phi}$ are the longitudinal electric and azimuthal 
magnetic fields on the wall surface. One of the first to use the surface impedance concept in the context of accelerator impedance calculations was Dohlus (see e.g., Ref. [7]). Known surface impedance models include dc [2], ac [1], and anomalous skin models of wall resistance [8], all of which can also be found in the classic paper of Reuter and Sondheimer [9]; other surface impedance models developed for accelerator physics applications are a model of wall roughness [10], and one for a pipe with small, periodic corrugations [11].

In the round case the resistive wall impedance and wakefield excited are well understood. The simplest "dc" model takes $\zeta$ to be

$$
\zeta(k)=\sqrt{\frac{k}{2 Z_{0} \sigma_{c}}}(1-i),
$$

with $\sigma_{c}$ the dc conductivity of the metallic walls. The more accurate "ac" model uses the same surface impedance Eq. (1), but with the dc conductivity replaced by $\tilde{\sigma}_{c}=\sigma_{c} /(1-i k c \tau)$, where $\tau$ is the so-called relaxation time of the metal. (Note that in LCLS-II the undulator beam pipe is made of aluminum, which has a relatively small relaxation time, resulting in ac and dc wakes and impedances that are similar.)

In the expressions for the longitudinal, dipole, and quadrupole impedances in flat geometry that we derive in this note, $\zeta$ is just an arbitrary function of $k$, and the same expressions can be used with surface impedances other than that of the resistive wall; for example, $\zeta$ can represent the case of two metallic plates with small corrugations. As numerical examples, however, we limit ourselves to the $\mathrm{dc}$ resistive wall model. One nice feature of this model is that the impedances and wakes can be written as universal functions involving the characteristic distance $s_{0}$, defined as

$$
s_{0}=\left(\frac{2 a^{2}}{Z_{0} \sigma_{c}}\right)^{1 / 3},
$$

where $a$ is the beam pipe radius. [For the LCLS-II undulator beam pipe, if we take the chamber half-height to be $a=2.5 \mathrm{~mm}$ and $\sigma_{c}=35 \mu \Omega^{-1} \mathrm{~m}^{-1}(\mathrm{Al})$, we obtain $s_{0}=9.8 \mu \mathrm{m}$.] For example, the longitudinal impedance in round geometry is given by [2]

$$
Z_{l}(\kappa)=2\left(\frac{s_{0}}{c a^{2}}\right)\left(\frac{2}{1-i} \frac{1}{\sqrt{\kappa}}-i \frac{\kappa}{2}\right)^{-1}
$$

where $\kappa=k s_{0}$; the longitudinal wake is also a universal function, of $s / s_{0}$, where $s$ is the distance the test particle trails the exciting particle. When the exciting particle moves slightly off axis, the transverse (dipole) impedance is excited, given by [2]

$$
Z_{y d}(\kappa)=4\left(\frac{s_{0}^{2}}{c a^{4} \kappa}\right)\left(\frac{2}{1-i} \frac{1}{\sqrt{\kappa}}-i \frac{\kappa}{2}\right)^{-1}
$$

Note that the function diverges at the origin as $\kappa^{-1 / 2}$.

\section{LONGITUDINAL IMPEDANCE}

We begin by deriving a general formula for the longitudinal impedance in flat geometry valid for high frequencies assuming a given surface impedance $\zeta(k)$. The material planes are located at $y= \pm a$, and the beam is at $x=x_{0}=0$ and $y=y_{0}(>0)$. We start by allowing the transverse positions of both the driving beam and the test particle to be arbitrary, giving us a generalized longitudinal impedance that we denote by $\tilde{Z}_{l}$. At the end we set the test particle position to $x=y=0$ and the driving particle position to $y_{0}=0$ to obtain the longitudinal impedance on axis, $Z_{l} \equiv \tilde{Z}_{l}\left(y_{0}=0, x=0, y=0\right)$.

The beam current density in frequency representation is $j_{z}=I_{\omega} \delta(x) \delta\left(y-y_{0}\right)$. Assuming frequency representation, the longitudinal electric field $E_{z}$ on the metal surface is related to $H_{x}$ by $E_{z}=\zeta H_{x}$. ${ }^{1}$ From Maxwell's equations it follows that $i k E_{z}=\partial H_{x} / \partial y$, so we can write the boundary condition at $y= \pm a$ as

$$
\frac{\partial H_{x}}{\partial y}=i k \zeta H_{x}
$$

One can derive from Maxwell's equations the following equation for $H_{x}$ :

$$
\frac{\partial^{2} H_{x}}{\partial x^{2}}+\frac{\partial^{2} H_{x}}{\partial y^{2}}=-\frac{4 \pi}{c}(\nabla \times \vec{j})_{x}=-\frac{4 \pi}{c} I_{\omega} \delta(x) \delta^{\prime}\left(y-y_{0}\right) .
$$

We can take the Fourier transform over $x$ to obtain

$$
\begin{aligned}
& \hat{H}_{x}(q)=\int_{-\infty}^{\infty} d x H_{x}(x) e^{i q x}, \\
& H_{x}(x)=\frac{1}{2 \pi} \int_{-\infty}^{\infty} d q \hat{H}_{x}(q) e^{-i q x} .
\end{aligned}
$$

In terms of $\hat{H}_{x}(q)$, Eq. (6) becomes

$$
-q^{2} \hat{H}_{x}+\frac{\partial^{2} \hat{H}_{x}}{\partial y^{2}}=-\frac{4 \pi}{c} I_{\omega} \delta^{\prime}\left(y-y_{0}\right) .
$$

This equation implies that $\hat{H}_{x}$ has a step discontinuity at $y_{0}$, $\hat{H}_{x}\left(y_{0}^{+}\right)-\hat{H}_{x}\left(y_{0}^{-}\right)=-4 \pi I_{\omega} / c$. The field can be written as

\footnotetext{
${ }^{1}$ The sign here is determined from the vector relation $\vec{E}_{t}=\zeta \vec{H}_{t} \times \vec{n}$, where $\vec{n}$ is directed inside the metal.
} 


$$
\begin{aligned}
\hat{H}_{x}^{ \pm}(y)= & \mp \frac{2 \pi}{c} I_{\omega} \cosh \left[q\left(y-y_{0}\right)\right] \\
& +A \sinh (q y)+B \cosh (q y),
\end{aligned}
$$

where $\hat{H}_{x}^{ \pm}(y)$ gives the field in the region $y \gtrless y_{0}$, and $A$ and $B$ are constants.

The boundary condition at $y= \pm a$ [Eq. (5)] yields

$$
\begin{aligned}
& q\left[-\frac{2 \pi}{c} I_{\omega} \sinh \left[q\left(a-y_{0}\right)\right]+A \cosh (q a)+B \sinh (q a)\right] \\
& =i k \zeta\left[-\frac{2 \pi}{c} I_{\omega} \cosh \left[q\left(a-y_{0}\right)\right]+A \sinh (q a)+B \cosh (q a)\right], \\
& q\left[-\frac{2 \pi}{c} I_{\omega} \sinh \left[q\left(a+y_{0}\right)\right]+A \cosh (q a)-B \sinh (q a)\right] \\
& =-i k \zeta\left[\frac{2 \pi}{c} I_{\omega} \cosh \left[q\left(a+y_{0}\right)\right]-A \sinh (q a)+B \cosh (q a)\right] .
\end{aligned}
$$

Solving these equations simultaneously we obtain

$$
\begin{aligned}
& A=\frac{2 \pi}{c} I_{\omega} \cosh \left(q y_{0}\right) \frac{q \sinh (q a)-i k \zeta \cosh (q a)}{q \cosh (q a)-i k \zeta \sinh (q a)}, \\
& B=-\frac{2 \pi}{c} I_{\omega} \sinh \left(q y_{0}\right) \frac{q \cosh (q a)-i k \zeta \sinh (q a)}{q \sinh (q a)-i k \zeta \cosh (q a)} .
\end{aligned}
$$

We can now find the generalized longitudinal impedance as $\tilde{Z}_{l}=-E_{z} / I_{\omega}$ :

$$
\tilde{Z}_{l}(k)=-\frac{1}{i k I_{\omega}}\left(\frac{\partial H_{x}}{\partial y}-\left.\frac{\partial H_{x}}{\partial y}\right|_{\zeta=0}\right),
$$

where the second term in the equation is needed to subtract out the vacuum field of the charge; the expression with $\zeta=0$ represents the case of perfectly conducting planes, where we know there is no longitudinal impedance. Combining Eqs. (9), (11), and (12), we obtain the result:

$$
\tilde{Z}_{l}(k)=\frac{2 \zeta}{c} \int_{-\infty}^{\infty} d q q \operatorname{csch}^{3}(2 q a) f(q) e^{-i q x},
$$

where $f(q)=n / d$, with

$$
\begin{aligned}
n= & q\left\{\cosh \left[q\left(2 a-y-y_{0}\right)\right]-2 \cosh \left[q\left(y-y_{0}\right)\right]\right. \\
& \left.+\cosh \left[q\left(2 a+y+y_{0}\right)\right]\right\}-i k \zeta\left\{\sinh \left[q\left(2 a-y-y_{0}\right)\right]\right. \\
& \left.+\sinh \left[q\left(2 a+y+y_{0}\right)\right]\right\}, \\
d= & {[q \operatorname{sech}(q a)-i k \zeta \operatorname{csch}(q a)][q \operatorname{csch}(q a)-i k \zeta \operatorname{sech}(q a)] . }
\end{aligned}
$$

[Note that the result is the same whether we take the upper or lower value of Eq. (9).] Equation (13) gives the general form of the longitudinal impedance, valid for any combination of $x, y, y_{0}$, within our geometric domain.

The longitudinal impedance $Z_{l}(k)$ is normally defined with the driving particle on the $y$ axis, and with the test particle following right behind it. From Eq. (13) we obtain

$$
\begin{aligned}
Z_{l}(k) & \left.\equiv \tilde{Z}_{l}(k)\right|_{x=y=y_{0}=0} \\
& =\frac{2}{c a} \int_{0}^{\infty} d x \frac{\operatorname{sech}(x)}{\cosh (x) / \zeta-i k a \sinh (x) / x}
\end{aligned}
$$

Note that the last expression, when applied to the dc rw impedance, was given in Ref. [5]; there the expression was derived from a more general result of Henke and Napoly [4], under the assumption that $s_{0} / a \ll 1$-which is true for normal metallic walls.

Using the dc resistive wall surface impedance, given in Eq. (1), the flat resistive wall impedance becomes

$$
\begin{aligned}
Z_{l}(\kappa)= & 2\left(\frac{s_{0}}{c a^{2}}\right) \int_{0}^{\infty} d x \operatorname{sech}(x) \\
& \times\left(\frac{2}{1-i} \frac{1}{\sqrt{\kappa}} \cosh (x)-i \kappa \frac{\sinh (x)}{x}\right)^{-1},
\end{aligned}
$$

where the normalized wave number $\kappa=k s_{0}$ and the characteristic distance $s_{0}$ is given by Eq. (2). We see that, as with the round case [Eq. (3)], the impedance is a universal function of $a$ and $s_{0}$. We numerically calculated $Z_{l}(k)$ using Eq. (16). In Fig. 1 we plot $\operatorname{Re}\left[Z_{l}(k)\right]$ (the solid curve); the dashed curve in the figure gives, for comparison, the result in a round beam pipe, with $a$ representing the pipe radius [given by the real part of Eq. (3)].

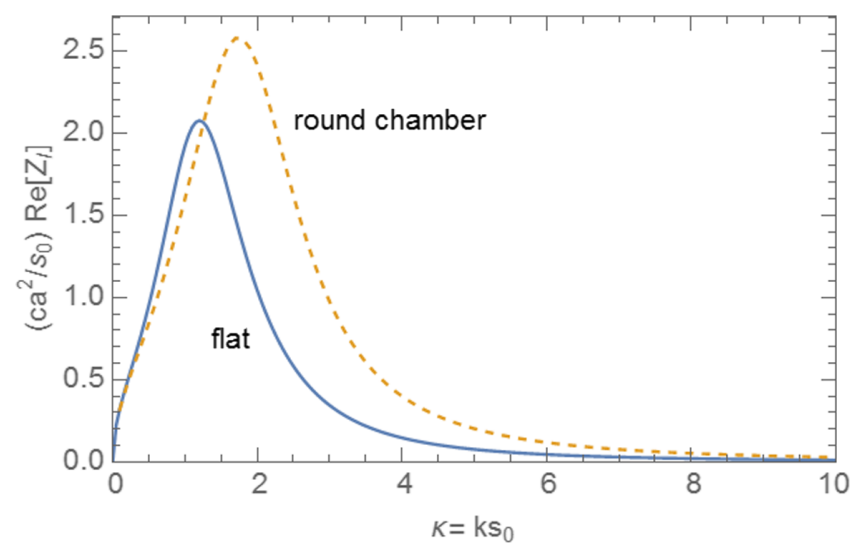

FIG. 1. Longitudinal resistive wall impedance on the axis between two parallel plates separated by distance $2 a$ (solid). Note that the abscissa gives the scaled frequency $\kappa=k s_{0}$ and the ordinate gives $\left(c a^{2} / s_{0}\right) \operatorname{Re}\left(Z_{l}\right)$. The round result, with $a$ the radius, is given by the dashed curve for comparison. 


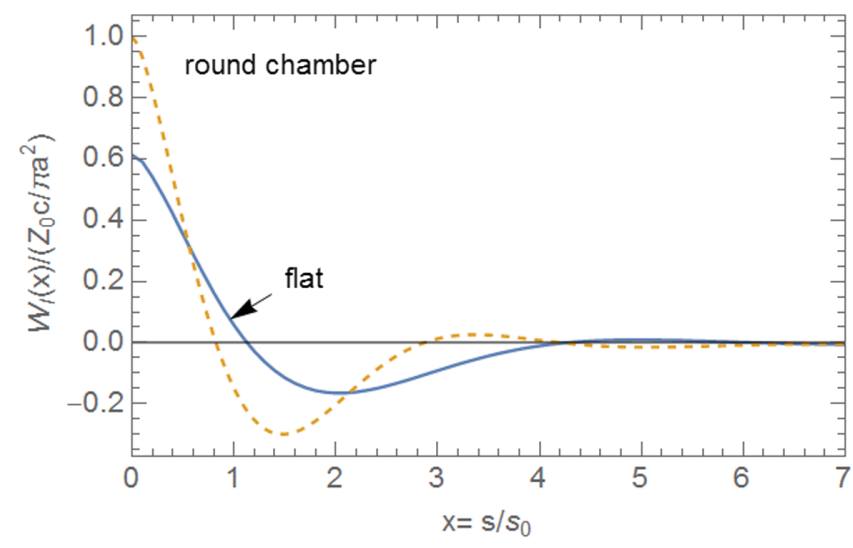

FIG. 2. Longitudinal resistive wall wake on the axis between two parallel plates separated by distance $2 a$ (solid). Note that the abscissa gives $x=s / s_{0}$ and the ordinate gives $W_{l} /\left(Z_{0} c / \pi a^{2}\right)$. The round result, with $a$ the radius, is given by the dashed curve for comparison.

From $\operatorname{Re}\left(Z_{l}\right)$ one can obtain the wake using the equation

$$
W_{l}(x)=\frac{2 c}{\pi s_{0}} \int_{0}^{\infty} \operatorname{Re}\left[Z_{l}(\kappa)\right] \cos \kappa x d \kappa
$$

where $x=s / s_{0}$. Again using the dc resistive wall surface impedance of Eq. (1), we obtain the longitudinal rw wake in flat geometry (see Fig. 2, the solid curve). Note that the value at the origin $W_{l}\left(0^{+}\right)=\left(\pi^{2} / 16\right)\left(Z_{0} c / \pi a^{2}\right)$. The dashed curve in the figure gives, for comparison, the wake in the round beam pipe, with $a$ representing the pipe radius.

\section{TRANSVERSE IMPEDANCE}

From the Panofsky-Wenzel theorem [12] the generalized transverse impedance is obtained from the generalized longitudinal one by

$$
\tilde{Z}_{y}=\frac{1}{k} \frac{\partial \tilde{Z}_{l}}{\partial y}
$$

Using Eq. (13) we find that

$$
\tilde{Z}_{y}(k)=-\frac{2 \zeta}{c k} \int_{-\infty}^{\infty} d q q^{2} \operatorname{csch}^{3}(2 q a) g(q) e^{-i q x}
$$

where $g(q)=n^{\prime} / d$, with

$$
\begin{aligned}
n^{\prime}= & q\left\{\sinh \left[q\left(2 a-y-y_{0}\right)\right]+2 \sinh \left[q\left(y-y_{0}\right)\right]\right. \\
& \left.-\sinh \left[q\left(2 a+y+y_{0}\right)\right]\right\}-i k \zeta\left\{\cosh \left[q\left(2 a-y-y_{0}\right)\right]\right. \\
& \left.-\cosh \left[q\left(2 a+y+y_{0}\right)\right]\right\}, \\
d= & {[q \operatorname{sech}(q a)-i k \zeta \operatorname{csch}(q a)][q \operatorname{csch}(q a)-i k \zeta \operatorname{sech}(q a)] . }
\end{aligned}
$$

However, like the normal longitudinal impedance, the normal transverse impedance is defined near the axis. In round geometry, if the driving current is slightly off axis, transversely the dipole impedance is excited. In flat geometry, for transverse driving and test positions near each other and near the $(y=0)$ symmetry plane, both dipole and quadrupole transverse impedances are excited, with the total impedances given by

$$
\tilde{Z}_{y}=y_{0} Z_{y d}+y Z_{y q}, \quad \tilde{Z}_{x}=\left(x_{0}-x\right) Z_{y q} .
$$

Here $x_{0}$ and $y_{0}(x$ and $y)$ are the offsets of the driving (test) particle, with $Z_{y d}\left(Z_{y q}\right)$ the vertical dipole (quadrupole) impedance. Note that the quad and dipole impedances are normalized to particle offset. Note also that the corresponding wake functions $\tilde{W}_{y}, W_{y d}, W_{y q}, \tilde{W}_{x}$, also satisfy the relations of the impedances, Eq. (21).

\section{A. The quadrupole impedance}

The quadrupole impedance is due to the distortion of the symmetric modes (those with nonzero $E_{z}$ on axis). It is antisymmetric and defocusing in $y$. For the calculation, we first put the driving particle on the axis in Eq. (19) to get

$\left.\tilde{Z}_{y}(k)\right|_{y_{0}=0}=\frac{1}{c k} \int_{-\infty}^{\infty} d q q e^{-i q x} \frac{\operatorname{sech}(q a) \sinh (q y)}{\cosh (q a) / \zeta-i k \sinh (q a) / q}$.

Then the vertical quad impedance is defined with the test particle at $x=0$ as a limit with small vertical offset:

$$
\begin{aligned}
Z_{y q}(k) & =\left.\lim _{y \rightarrow 0} \frac{1}{y} \tilde{Z}_{y}(k)\right|_{x=y_{0}=0} \\
& =\frac{2}{c k a^{3}} \int_{0}^{\infty} d x x^{2} \frac{\operatorname{sech}(x)}{\cosh (x) / \zeta-i k a \sinh (x) / x} .
\end{aligned}
$$

We have numerically calculated the quad impedance in flat geometry using as surface impedance that of the dc rw wall [Eq. (1)]. The product $\sqrt{\kappa} R e\left(Z_{y q}\right)$, is plotted as function of $\kappa=k s_{0}$ in Fig. 3 (the green curve). We see that the asymptote near the origin is given by

$$
\operatorname{Re}\left[Z_{y q}(\kappa)\right]=\frac{\pi^{2}}{12}\left(\frac{s_{0}^{2}}{c a^{4}}\right) \kappa^{-1 / 2} .
$$

From the real part of a transverse impedance, like $\operatorname{Re}\left(Z_{y q}\right)$, one can obtain the wake from the sine transform:

$$
W_{y q}(x)=\frac{2 c}{\pi s_{0}} \int_{0}^{\infty} \operatorname{Re}\left[Z_{y q}(\kappa)\right] \sin \kappa x d \kappa,
$$




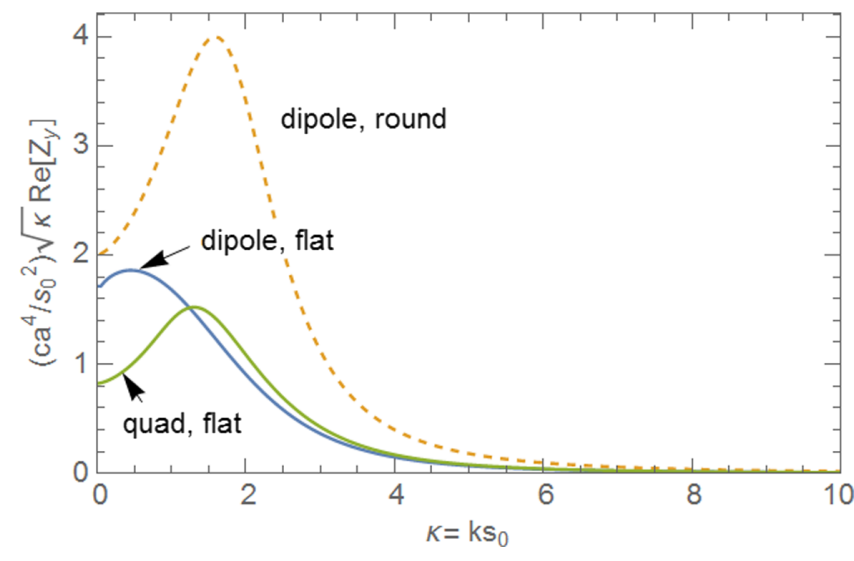

FIG. 3. Transverse resistive wall impedance near the axis between two parallel plates separated by distance $2 a$, showing $\operatorname{Re}\left(Z_{y d}\right)$ (blue) and $\operatorname{Re}\left(Z_{y q}\right)$ (green). Note that the abscissa gives the scaled frequency $\kappa=k s_{0}$ and the ordinate gives $\left(c a^{4} / s_{0}^{2}\right) \sqrt{\kappa} \operatorname{Re}\left(Z_{y}\right)$. The round result, with $a$ the radius, is given by the dashed curve for comparison.

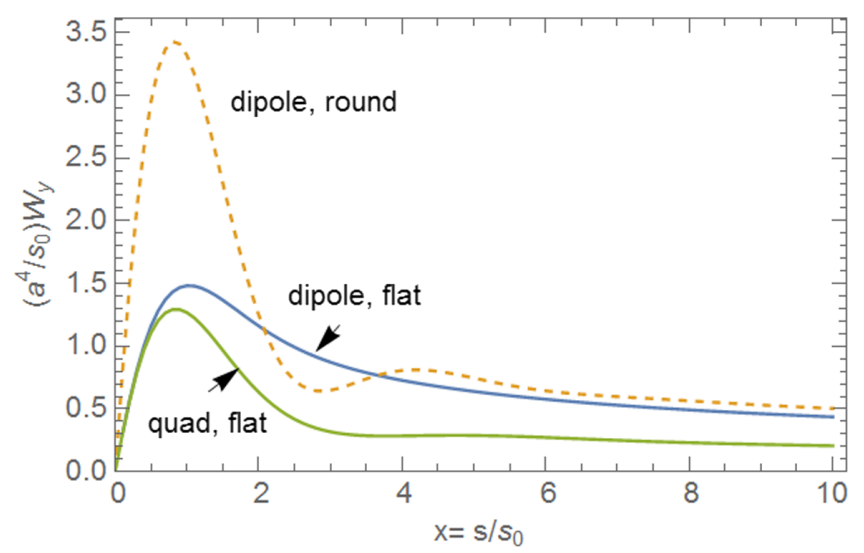

FIG. 4. Transverse resistive wall wake between two parallel plates separated by distance $2 a$, showing $W_{y d}$ (blue) and $W_{y q}$ (green). Note that the abscissa gives $x=s / s_{0}$ and the ordinate gives $\left(a^{4} / s_{0}\right) W_{y}$. The round result, with $a$ the radius, is given by the dashed curve for comparison.

where $x=s / s_{0}$. The quad wake in flat geometry is shown in Fig. 4 (the green curve). Note that the slope at the origin

$$
\frac{d W_{y q}}{d s}\left(0^{+}\right)=\frac{2 Z_{0} c}{\pi a^{4}}\left(\frac{\pi}{4}\right)^{4}
$$

and that the peak is located near $s=s_{0}$.

\section{B. The dipole impedance}

The dipole impedance is due to the offset of the driving charge. To obtain it we first put the test particle at $x=0$ and on axis in Eq. (19):

$$
\left.\tilde{Z}_{y}(k)\right|_{x=y=0}=\frac{2}{c k} \int_{0}^{\infty} d q q \frac{\operatorname{csch}(q a) \sinh \left(q y_{0}\right)}{\sinh (q a) / \zeta-i k \cosh (q a) / q} .
$$

Then the vertical dipole impedance is defined as a limit for small offset of the driving particle (at $y=y_{0}$ ) as

$$
\begin{aligned}
Z_{y d}(k) & =\left.\lim _{y_{0} \rightarrow 0} \frac{1}{y_{0}} \tilde{Z}_{y}(k)\right|_{x=y=0} \\
& =\frac{2}{c k a^{3}} \int_{0}^{\infty} d x x^{2} \frac{\operatorname{csch}(x)}{\sinh (x) / \zeta-i k a \cosh (x) / x} .
\end{aligned}
$$

We have numerically calculated the dipole impedance in flat geometry using as surface impedance that of the dc rw wall [Eq. (1)]. The real part of the dipole impedance, $\operatorname{Re}\left(Z_{y d}\right)$, is plotted in Fig. 3 (the blue curve), where the dipole wake for a round pipe [dashed; given by the real part of Eq. (4)] is also given for comparison. We see that, like the quad wake, $\operatorname{Re}\left(Z_{y d}\right)$ varies as $\kappa^{-1 / 2}$ near the origin, but with an asymptote that is twice as large,

$$
\operatorname{Re}\left[Z_{y d}(\kappa)\right]=\frac{\pi^{2}}{6}\left(\frac{s_{0}^{2}}{c a^{4}}\right) \kappa^{-1 / 2} .
$$

From the real part of a transverse impedance one can again obtain the wake from a sine transform, see Fig. 4 (the blue curve). Note that the slope at the origin is the same as we found for the quad wake. The sum of the two slopes is $2(\pi / 4)^{4}=0.76$ times the size of the slope of the dipole wake in a round pipe (with $a$ the beam pipe radius).

\section{Application to LCLS-II undulator beam pipe}

The longitudinal impedance formula, Eq. (15), has previously been applied to LCLS-II undulator parameters, taking the surface impedance to represent wall resistance and roughness [10], and recently to represent wall resistance at cryogenic temperatures, by using an anomalous skin effect surface impedance [8].

The effects of the transverse impedance in the LCLS-II undulator beam pipe have been characterized as weak, because the bunch is very short. However, the new formulas allow one to quantify the effects. In Ref. [13], using these formulas, it is shown that-even for the long bunch, large charge $(300 \mathrm{pC})$ option - the quad and dipole wake effects in the undulator of LCLS-II are negligibly small.

\section{CONCLUSIONS}

In this paper, beginning with Maxwell's equations and assuming only that the wall interaction can be approximated by a surface impedance, we have derived formulas for the generalized longitudinal and transverse impedance in flat geometry, where by generalized we mean that the 
(transverse) positions of the driving and test particle trajectories can be located anywhere within the region between the two plates; from these, the corresponding point charge wakefields can also be obtained. Then, from the generalized impedance formulas, by limiting the driving and test particle trajectories to be near each other and near the axis, we have obtained the normal longitudinal, dipole, and quad impedances in flat geometry. These equations can be applied to any surface impedance, such as the known dc, ac, and anomalous skin models of wall resistance, a model of wall roughness, and a model for a pipe with small corrugations.

In this note, we have shown that-for the particular case of dc wall resistance - the longitudinal impedance obtained here agrees with a result found in the literature, a result that was originally derived from a very general formula by Henke and Napoly. We have here, in addition, produced plots of the longitudinal and transverse impedances and wakes for the case of dc wall resistance.

\section{ACKNOWLEDGMENT}

We thank J. Welch for explaining to us about various aspects of the undulator region of LCLS-II. This work was supported by Department of Energy Contract No. DEAC02-76SF00515.

[1] K. Bane and M. Sands, The short-range resistive wall wakefields, AIP Conf. Proc. 367, 131 (1996).

[2] A. Chao, Physics of Collective Beam Instabilities in High Energy Accelerators (J. Wiley \& Sons, New York, 1993), Chap. 2.
[3] A. Piwinski, Wake fields and ohmic losses in flat vacuum chambers, DESY HERA Report No. 92-04, 1992.

[4] H. Henke and O. Napoly, in Proceedings of the 2nd European Particle Accelerator Conference, France, 1990 (Atlantica-Séguier, Paris, 1990), pp. 1046-1048.

[5] K. Bane and G. Stupakov, Resistive wall wakefield in the LCLS undulator, in Proceedings of the 21st Particle Accelerator Conference, Knoxville, TN, 2005 (IEEE, Piscataway, NJ, 2005), pp. 3390-3392.

[6] A. Piwinski, Wake fields and ohmic losses in round vacuum chambers, DESY HERA Report No. 92-11, 1992.

[7] M. Dohlus, Impedance of beam pipes with smooth shallow corrugations, DESY TESLA Report No. 2001-26, 2001.

[8] G. Stupakov et al., Resistive wall wakefields of short bunches at cryogenic temperatures, Phys. Rev. ST Accel. Beams (in press).

[9] G. E. H. Reuter and E. H. Sondheimer, The theory of the anomalous skin effect in metals, Proc. R. Soc. A 195, 336 (1948).

[10] K. Bane and G. Stupakov, Roughness tolerance studies for the undulator beam pipe chamber of LCLS-II, 27th Linear Accelerator Conference, Geneva, Switzerland, 2014 (CERN, Geneva, 2014);Report No. LCLS-II-TN-14-06, 2014.

[11] G. Stupakov and K. L. F. Bane, Surface impedance formalism for a metallic beam pipe with small corrugations, Phys. Rev. ST Accel. Beams 15, 124401 (2012).

[12] W. Panofsky and W. Wenzel, Some considerations concerning the transverse deflection of charged particles in radiofrequency fields, Rev. Sci. Instrum. 27, 967 (1956).

[13] K. Bane and G. Stupakov, Using surface impedance for calculating wakefields in flat geometry, Reports No. LCLS-II-TN-15-02 and No. SLAC-PUB-16203, 2015. 